\title{
PERCEPCIÓN SOCIAL DEL GRAN PREMIO DE EUROPA DE FÓRMULA UNO ENTRE LOS RESIDENTES DEL MUNICIPIO DE VALENCIA
}

\section{SOCIAL PERCEPTIONS OF THE FORMULA ONE EUROPEAN GRAND PRIX AMONG RESIDENTS OF VALENCIA CITY}

\author{
Duclos Bastías, Daniel \\ Unidad de Investigación del Rendimiento Físico y Deportivo, Departamento de Educación Física y Deportiva. \\ Universidad de Valencia.
}

DUCLOS B.D. Percepción Social del Gran Premio de Europa de Fórmula Uno entre los residentes del Municipio de Valencia. Mot.Hum. 13(2); 118-122: 2012.

\section{RESUMEN}

Los objetivos principales de la presente investigación han sido, analizar la percepción que los residentes de Valencia tienen sobre la realización del Gran Premio de Europa (GPE) de Fórmula Uno en la ciudad: conocimientos, intereses, actitudes, comportamientos y expectativa de dicha población, con respecto a este evento deportivo. Se ha aplicado un cuestionario estructurado de 29 ítems a una muestra de residentes del municipio de Valencia compuesta por 421 sujetos. Se ha obtenido un error de muestreo de $\pm 4,9 \%$. Las conclusiones informan que en general la población valenciana pose conocimiento sobre el evento deportivo. Sin embargo han manifestado tener escasa ilusión por la celebración del evento en la ciudad. La muestra percibe que los sectores más beneficiados con el desarrollo del evento, serían el Turismo y la Economía. También se informa que el colectivo menos beneficiado serían los Ciudadanos. La gran mayoría de consultados ha indicado que no asistirá al GPE de Fórmula Uno.

Palabras clave: Eventos, organización Fórmula Uno, repercusión socio-económica, imagen, percepción social.

\begin{abstract}
The main objectives of this research have been, to analyze the perception that residents of Valencia have on the realization of the European Grand Prix Formula One in the city: knowledge, interests, attitudes, behaviors and expectations of the population, to respect to this event. It has implemented a structured questionnaire of 29 items to a sample of residents of the municipality of Valencia consists of 421 subjects. It has obtained a sampling error of $\pm 4.9 \%$. The findings report that in general the population of Valencia pose knowledge about the event. But have expressed lack of enthusiasm for the celebration of the event in the city. The sample perceived that the most benefit from the development of the event would be the tourism and economy. It is also reported that the group less benefited would be the citizens. The vast majority of answers indicated they will not attend the European Grand Prix Formula One.
\end{abstract}

Key Words: Events, Formula One, organization, socio-economic impact, image, social perception. 


\section{INTRODUCCIÓN}

El deporte se ha ofrecido como medio de entretenimiento en el tiempo de ocio a lo largo de la historia.

En las sociedades contemporáneas se ha consolidado como fenómeno cultural característico. En cinco décadas el posicionamiento global del deporte espectáculo ha sido inminente, y es que los grandes eventos continúan teniendo un gran nivel de aceptación popular y audiencias muy notorias en la televisión y otros medios de comunicación, (1). Además el creciente atractivo por parte de las ciudades o países de acoger grandes eventos deportivos a menudo se convierte en una ardua tarea por hacerse con los derechos de organización de acontecimientos de una edición, como los Juegos Olímpicos o la Copa del Mundo de Fútbol, u otros eventos con concesiones por un determinado número de años, tal y como sucede con los masters de tenis o los grandes premios de Motociclismo o de Automovilismo. Este interés, evidentemente pasa en general por una cuestión económica y en particular por la promoción del turismo y oportunidades de empleo, $(2,3,4,5)$.

La medición objetiva de la percepción social de los eventos deportivos, es un área de investigación importante, que contribuye significativamente a la mejora de la planificación y la gestión de eventos futuros.

En el contexto local, la ciudad de Valencia en los últimos años, ha albergado la Copa América de Vela, además del Campeonato del Mundo IAAF de Atletismo Indoor. Desde 1999 en el circuito Ricardo Tormo se organiza el Gran Premio del Mundial de Motociclismo y desde el año 2008 se lleva a cabo el GPE de Fórmula Uno en el circuito urbano emplazado en la zona del puerto de la ciudad, como también cabe señalar la celebración de dos Copas del Rey de Fútbol y el Open 500 de Tenis que este año alberga su quinta edición.

El presente artículo tiene como fin informar sobre el análisis de los datos obtenidos sobre de la percepción que los residentes de Valencia tienen sobre la realización del GPE de Fórmula Uno en la ciudad: conocimientos, intereses, actitudes, y expectativas de dicha población, con respecto a este evento deportivo.

\section{MATERIAL Y MÉTODOS}

\section{Población objeto de estudio}

La población objeto de estudio está compuesta por las personas que cumplieron con las siguientes características: hombres y mujeres, residentes del municipio de Valencia de edad comprendida entre 15 y 65 años. Esto ha supuesto una población total de 561.035 residentes según los datos registrados en el padrón municipal del año 2008, (6).

\section{Muestra}

Se ha obtenido una muestra de 421 sujeto: 235 hombres $(55,8 \%)$ y 186 mujeres $(44,2 \%)$, no habiendo contestado a esta pregunta 2 sujetos $(, 5 \%)$. El error de muestral se ha ubicado en $\pm 4,9 \%$, con un nivel de confianza del $95 \%(\mathrm{p}=\mathrm{q}=50)$ para toda la muestra en su conjunto.

\section{Procedimiento, recogida y análisis de los datos}

El procedimiento de muestreo seguido ha sido de tipo intencional (no probabilístico). El tamaño de la muestra ha sido distribuido en 19 puntos de muestreo los cuales han sido elegidos considerando la organización distrital municipal, manteniendo el principio de proporcionalidad.

Los datos recogidos fueron introducidos, tabulados y tratados mediante el paquete estadístico SPSS versión 17.0. Se efectúo un análisis descriptivo de las variables analizadas en el cuestionario incluyendo estadísticos como la media, las frecuencias y la desviación típica para el análisis e interpretación de los datos. Para analizar las diferencias en función de las variables de sexo y edad se efectúo un análisis diferencial utilizando la prueba chi-cuadrado de Pearson (ítems nominales), la prueba $\mathrm{T}$ para muestras independientes $\mathrm{y}$ ANOVA con la aplicación previa de la prueba de Levene para comprobar la homocedasticidad de las distintas variables. 


\section{Instrumento}

Esta investigación ha utilizado como técnica de recogida de información la entrevista estandarizada, mediante cuestionario, el cual ha sido elaborado, validado y aplicado por el equipo de investigadores del estudio «Conocimiento e identificación con los Juegos Mediterráneos (JJ.MM) por parte de la población de Almería y provincia. Evolución durante los 4 años anteriores al evento», llevado a cabo por un equipo de investigadores de la Universidad de Almería y el Comité Organizador de los XV JJ.MM de Almería 2005, (7).

Por tratarse del mismo objeto de estudio, población que alberga un gran evento deportivo internacional, en el caso de la presente investigación, el GPE de Fórmula Uno de Valencia, y con el objetivo de mejorar la aplicación del cuestionario y facilitar la obtención de los resultados en función de los objetivos de la investigación, un equipo de investigadores de la UIRFIDE de la Universidad de Valencia, adaptó y estableció las correspondientes modificaciones al cuestionario original considerando aquellas características del contexto en particular en que se desarrolló esta investigación. Una vez realizadas las modificaciones pertinentes el cuestionario definitivo contó con 29 ítems agrupados en 5 bloques temáticos:

1.- Conocimiento e identificación con el Gran Premio de Fórmula Uno de Valencia. 2.- Beneficios que reportará el Gran Premio de Fórmula Uno en Valencia. 3.- Gestión y organización del Gran Premio de Fórmula Uno en Valencia. 4.- Hábitos deportivos. 5.- Variables Sociodemográficas.

\section{RESULTADOS}

Un 95,5\% de la muestra afirma que el GPE de Fórmula Uno es una competición deportiva internacional.

Un $43 \%$ de los entrevistados percibe que el evento tiene poca y ninguna importancia a nivel social. En tanto, un $62 \%$ entiende que existe bastante y mucha importancia nivel deportivo. La importancia a nivel económico recibe una valoración de $53 \%$ de los encuestados que le otorgan bastante y mucha importancia, un 13,8\% señala algo de importancia, mientras que el $31,1 \%$ valora como ninguna y poca importancia en esta materia. Para este último ítem se han encontrado diferencias estadísticamente significativas entre los grupos de edad: Joven $(3,48)$, Adulto-Joven $(3,40)$, Adulto $(3,25)$ y +Adulto $(2,82)$ con valores $\mathrm{F}=3,034$ y $\mathrm{Sig}=, 029 *$ (* $\mathrm{p} \leq 0,05)$.

Respecto al grado de información sobre el GPE de Fórmula Uno de Valencia, los ciudadanos indican tener ninguna y poca, expresada por un $38,1 \%$. Por el contrario sólo un $27 \%$ de los encuestados manifiesta tener bastante y mucho grado de conocimiento. Por otra parte el $34,3 \%$ de entrevistados que expresa tener algo de conocimiento sobre el Gran Premio de Fórmula Uno de Valencia. Se han encontrado diferencias estadísticamente significativas entre el grupo de Hombres $(3,16)$ y de Mujeres $(2,45)$ con valores $\mathrm{F}=43,270$ y $\mathrm{Sig}=, 000 * * *(* * * \mathrm{p} \leq 0,001)$.

Un 69,2\% de los ciudadanos de Valencia tienen ninguna y poca ilusión por este acontecimiento. En tanto para el $31,1 \%$ restante de encuestados tiene bastante y mucha ilusión por la realización del evento deportivo. Se han encontrado diferencias estadísticamente significativas entre el grupo de Hombres $(2,13)$ y el de Mujeres $(1,74)$ con valores $\mathrm{F}=17,760$ y $\mathrm{Sig}=, 000 * * *(* * * \mathrm{p} \leq 0,001)$.

En relación a cómo los encuestados perciben que el resto de la sociedad se identifica con dicho acontecimiento deportivo, independientemente de que compartan la opinión, un $64,7 \%$ de la muestra indica nada y poca identificación, mientras un $35,2 \%$ estima que esta es bastante y mucha.

Para la población entrevistada, el sector que se verá más beneficiado con la realización del GP de Fórmula Uno en la ciudad será el turismo. De este modo, un $76,9 \%$ cree que el sector turístico se beneficiará bastante y mucho. La economía también se beneficiará bastante y mucho en opinión de un $63,5 \%$ de la muestra. En esta misma línea, los encuestados manifiestan que aspectos como el urbanismo e instalaciones deportivas tendrían escaso beneficio, así lo indica el $69 \%$ de los entrevistados que afirman que el grado de beneficio sería nada y poco. Finalmente otros aspectos donde se pueden observar una diferencia porcentual más estrecha son en infraestructura general, donde el $59,1 \%$ de la muestra valora como nada y poco de 
beneficio, versus un $40,8 \%$ que valora los beneficios como bastante y mucho. De igual manera se puede observar en el ítem que mide el beneficio del empleo que un $55,6 \%$ de los consultados asegura que existe escaso beneficio (nada y poco), mientras que un $44,4 \%$ considera que habrá bastante y mucho beneficio en este sector.

Por el contrario un $76 \%$ de los consultados percibe que el grado de beneficio de la práctica deportiva con la celebración del evento sería poco y nada.

En relación a la percepción que tienen los residentes entrevistados respecto al grado de utilización que tendría el circuito de Fórmula Uno después de la celebración del GPE, un $84,1 \%$ ha indicado que el grado de utilización será ninguno y poco. Se han encontrado diferencias estadísticamente significativas entre los grupos de edad: Joven $(1,90)$, Adulto-Joven $(1,65)$, Adulto $(1,52)$ y +Adulto $(1,52)$ con valores $\mathrm{F}=2,696$ y $\mathrm{Sig}=, 046^{*}$ $(* \mathrm{p} \leq 0,05)$.

Finalmente se preguntó a la muestra por la asistencia al GPE de Fórmula Uno en Valencia. Ante esta cuestión, el 83,3\% afirma que no asistirá al evento. Mientras que el 16,7\% restante aseguró que si lo hará. Se han encontrado diferencias estadísticamente significativas entre las frecuencias del grupo de Hombres y el de Mujeres con valores $\mathrm{x}^{2}=6,706$ y Sig $=, 010 * *(\mathrm{p} \leq 0,05)$.

\section{DISCUSIÓN}

En comparación los estudios, la presente investigación informa de resultados en la misma línea, respecto a la identificación del tipo de evento. Esto puede ser confirmado por el estudio (8) que informa de una elevada frecuencia de audiencia de programas deportivos que se emiten por televisión (fuente de representación social más potente) un 77 $\%$ de los españoles (y un $78 \%$ de los valencianos) que ve con frecuencia este tipo de programación.

En relación a la importancia social que los ciudadanos conceden al evento, los resultaos de los estudios (7) y (9), otorgan bastante y mucha importancia a los JJ.MM con un $69,7 \%$ y un $60 \%$ respectivamente. En tanto, los resultados del presente estudio rebajan sustancialmente ese nivel, como lo afirma un $43 \%$ con ninguna y poca importancia social. Sin embargo, en la importancia sobre el ámbito deportivo, los resultados en los estudios (7) y (9) son similares. Los ciudadanos consultados valoran como bastante/mucho esta importancia con $76,7 \% 75,2 \%$ respectivamente. En el presente trabajo, la

importancia deportiva otorgada al evento en comparación con los datos señalados es un poco más baja, pero mantiene niveles altos, un $62 \%$ de la muestra otorga bastante y mucha importancia al evento.

En relación a la importancia económica del evento, los resultados de los estudios (7) y (9) conceden altas valoraciones, así lo manifiestan el $76,9 \%$ y un $58,7 \%$ otorgan bastante y mucha importancia económica a los JJ.MM.

Otros estudios que han indagado sobre el impacto social de competiciones de deporte motor, y que aportan información valiosa en relación al impacto o repercusión del evento, (10) analizó las reacciones de la comunidades anfitrionas de grandes eventos de motor como el Gran Premio (GP) de Australia de Fórmula Uno celebrado en la ciudad de Melbourne y la Indy Car en la ciudad de Gold Coast. Los resultados de la tesis informan que la muestra percibe que ambos acontecimiento impactan a nivel internacional, lo cual supone beneficio para las ciudades. La muestras de Melbourne $(90,6 \%)$ y Gold Coast $(96,6 \%)$ afirman que este tipo de eventos aumenta el posicionamiento internacional de la ciudad, mientras que las muestras de el estudio (10) y la analizada en la presente investigación otorgan repercusión internacional (47,3\% y 53,9\% respectivamente.

Analizando los resultados obtenidos por el estudio dirigido por $(7,9)$, en relación a la información que se tiene sobre el evento un $66,2 \%$ y un $55,1 \%$ (para cada muestra respectiva) de los entrevistados han afirmado tener ninguna, poca y algo de información sobre el evento.

En el estudio (11) que analiza la percepción de los residentes de la localidad de Macao (China) sobre la celebración del GP de Fórmula 3000 celebrado en esa ciudad, los resultados informan que los ciudadanos manifiestan estar de acuerdo en que la celebración del evento aumenta el orgullo 


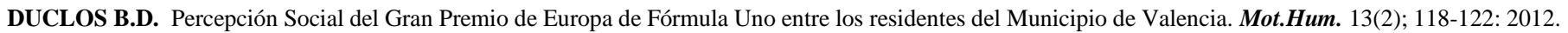

ciudadano, esto lo afirmó un $69,7 \%$ de los entrevistados. En relación a esto el estudio (10) ha encontrando valores más bajos en las muestras que analizó de Melbourne y Gold Coast con un 44,6\% y $56,1 \%$. Además otros datos reseñables de este último trabajo son los referidos al grado en que estos eventos afectan el estado de ánimo de los ciudadanos. En este sentido las muestras de Melbourne y de Gold Coast para el 78,6\% y el $76,4 \%$ (respectivo) el estado de ánimo de la gente no cambia.

Por otra parte, ante el positivo apoyo y alto orgullo de los ciudadanos de Macao por acoger el GP de Fórmula 3000, las investigación $(11,12)$ explican, que estos altos porcentajes (alto orgullo) se pueden atribuir a las diferencias culturales y factores políticos propios de la cultura china.

Respecto al grado de identificación que tiene la población residente de Valencia con el GPE de Fórmula Uno, la mayoría de los entrevistados, afirma que esta es escasa, como así lo indica un $64,7 \%$ (nada y poco). Este dato es confirmado si interpretamos los datos recogidos en el estudio (8) en el que informan que los valencianos manifiestan un bajo interés $(23 \%)$ hacía el deporte espectáculo, lo cual se puede interpretar como una baja identificación con este tipo de actuaciones.

\section{REFERENCIAS BIBLIOGRÁFICAS}

1. Duclos, D., Añó, V., \& Pablos, C. Percepción social del gran premio de Europa de Fórmula 1 entre los ciudadanos de Valencia. Motricidad European Journal of Human Movement. 2010; 17 (25): 143-164.

2. Añó, V. Organización y Gestión de Actividades Deportivas. Los Grandes Eventos. Barcelona: Inde; 2003.

3. Añó, V. Organización de Eventos y Competiciones Deportivas. Valencia: Universitàt de València; 2011.

4. Añó, V. Organización de Grandes Eventos Deportivos Internacionales. Arbor. 2000; 650: 265-287.

5. Añó, V. Beneficios Socioeconómicos de la Organización de Grandes Eventos Deportivos. Impacto sobre la Economía y el Turismo. Nuevas Aportaciones al estudio de la Actividad Física y el Deporte. Universitàt de València. 2001:15-31.
6. Instituto Valenciano de Estadística. Padrón Municipal [base de datos Internet]. Valencia: Mayo 1990 [Marzo Setiembre 2008; Octubre 2009]. http://www.ive.es.

7. Casimiro, A., \& Añó, V. Imagen Social de los Juegos Mediterráneos en la sociedad almeriense durante el año 2004. Comparativa con años 2002 y 2003. Almería: Universidad de Almería; 2005.

8. García Ferrando, M., Mestre, J. \& Llopis, R. Los valencianos y el Deporte. Valencia: Ayuntamiento de Valencia; 2007.

9. Añó, V., Pablos, C. \& Calabuig, F. Opinión de los Tarraconenses sobre la candidatura a los Juegos Mediterráneos de 2017. Valencia: Universitat de València; 2009

10. Fredline, E. Host community reactions to major sporting events: The Gold Coast Indy and the Australian Formula One Grand Prix in Melbourne. [tesis doctoral]. Gold Coast: Griffith University; 2000.

11. Zhou, Y. Resident's Perceptions towards the Impacts of the Macao Grand Prix. Journal of Convention \& Event Tourism. 2010; 11(2): 138-153.

12. Zhou, Y. \& Ap, J. Resident's perceptions towards the impacts ofthe Beijing 2008 Olympic Games. Journal of Travel Research. 2009; 48(1): 78-91.

\section{Dirigir Correspondencia a:}

Daniel Duclos Bastías.

Aulari V Calle Gascó Oliag, 3

46010 Valencia (ESPAÑA).

dadubas@alumni.uv.es

RECIBIDO: 16-04-2012

ACEPTADO: 13-12-2012 